Original Article

\title{
Investigation of the effect of REM sleep deprivation on epileptic seizures caused by pentylenetetrazole in mice
}

\author{
Recep Akkaya $^{1}$ (D) Sebahattin Karabulut ${ }^{2}$ - Ahmet Sevki Taskiran ${ }^{3}$ \\ ${ }^{1}$ Department of Biophysics, Sivas Cumhuriyet University, School of Medicine, Sivas, Turkey \\ ${ }^{2}$ Department of Medical Services and Techniques, Sivas Cumhuriyet University, Vocational School of Health Services, \\ Sivas, Turkey \\ ${ }^{3}$ Department of Medical Physiology, Sivas Cumhuriyet University, School of Medicine, Sivas, Turkey
}

\section{ABSTRACT}

\begin{abstract}
Aim: To investigate whether different periods of rapid eye movement sleep deprivation (REM SD) contribute to seizure susceptibility, hippocampal oxidative status and balance of inhibition-excitation in the acute epilepsy model.
\end{abstract}

Methods: REM SD was performed using the modified multiple platforms method on adult male BALB/c mice. Pentylentetrazol (PTZ) was injected to induce seizures and hippocampal total antioxidant status (TAS), total oxidant status (TOS), gamma aminobutyric acid (GABA), and glutamate levels were measured using the ELISA method.

Results: PTZ-induced seizures following $8 \mathrm{~h}$ and $72 \mathrm{~h}$ REM SD significantly reduced the hippocampal TAS levels, but did not affect the TOS levels. In REM SD groups, especially after 8 hours of REM sleep loss, there was a significant increase in glutamate in PTZ induction. The hippocampal GABA levels were increased by PTZ-induced seizures after $72 \mathrm{~h}$ REM SD. PTZinduction after 8 hours of RAM SD leads to a significant increase in the seizure duration.

Conclusion: It can be speculated that the REM SD can contribute to seizure susceptibility by changing the oxidant-antioxidant balance and excitatory and inhibitory tone in the hippocampus.

Keywords: REM sleep deprivation, hippocampus, seizure susceptibility, oxidative stress.

(C) 2020 experimentalbiomedicalresearch.com

$\triangle$ Dr. Recep Akkaya

Department of Biophysics, Sivas Cumhuriyet University,

School of Medicine, Sivas, Turkey

E mail: recepakkaya5835@gmail.com

Received: 2020-02-19 / Revisions: 2020-03-03

Accepted: 2020-03-09 / Publication Date: 2020-06-13

\section{Introduction}

Epilepsy is one of the most common neurological disorders characterized by recurring seizures that originated due to imbalances in excitation and inhibition in the brain [1,2]. Despite the presence of existing antiepileptic drugs (AEDs) that can successfully prevent recurrent seizures in the majority of patients, about a third of patients are resistant to treatment [3]. Also, AEDs do not affect the underlying pathophysiology and progression of the disease, they often provide symptomatic therapy [4]. Current studies aim to elucidate the cellular mechanisms in which a 
normal brain is epileptic, that is, epileptogenesis. This term refers to a process that progressively alters neuronal excitability. The development of antiepileptogenic drugs that prevent or reduce the progression of the disease could have a major effect on the life of patients with epilepsy. Therefore, understanding the cellular mechanisms of epileptogenesis and its treatment are research priorities on the political agendas in both Europe and the United States [5].

Sleep loss is widespread in neurodegenerative diseases and pieces of evidence indicate that sleep interacts with disease and is not only a symptom of it [6]. It has been reported that sleep deprivation (SD) can alter cortical excitability, which is seen as the balance between the inhibitor and excitatory of neuronal circuits in the cortex, which can reduce the epileptic threshold [7]. Interestingly, the seizures are less common in rapid eye movement (REM) sleep, so a seizure-protecting role has been proposed for this sleep phase [8]. Although SD is thought to promote neurodegeneration, not much is known about how they interact mutually. Therefore, in this study, we aimed to investigate whether different periods of REM SD contribute to seizure susceptibility. For this purpose, we evaluated epileptic behaviors induced by pentylentetrazol (PTZ) and examined the oxidative status and inhibition-excitation balance in the hippocampus.

\section{Materials and Methods}

The study was performed with 24 adult male, weighing 35-38 g, BALB-c Albino mice (obtained from Cumhuriyet University Animal Laboratory). Five mice per cage were housed under controlled environmental conditions with regard to temperature $\left(23 \pm 2^{\circ} \mathrm{C}\right)$, humidity (35\%-60\%), and a 12:12 h light-dark cycle.
The mice were allowed access to food water and libitum. All procedures were performed in accordance with the guidelines of the Local Ethics Committee for the welfare of experimental animals (Registry Number: 65202830-050.04.04-166 dated 09.04.2018). All efforts were made to decrease the number of animals used to a minimum. The procedures were conducted according to routine animal care guidelines, and all experimental procedures complied with the Guide for the Care and Use of Laboratory Animals (1996).

\section{Experimental procedure}

The mice were randomly assigned to into four groups each containing six animals as follow: The control group, not deprived of REM sleep; the PTZ group, PTZ injected; the REM SD 8+PTZ group, PTZ injected after deprived of REM sleep for $8 \mathrm{~h}$; the REM SD $72+\mathrm{PTZ}$ group, PTZ injected after deprived of REM sleep for $72 \mathrm{~h}$.

\section{REM SD procedure}

The modified multiple platform method was used to restrain REM sleep for $8 \mathrm{~h}$ and $72 \mathrm{~h}$ [9]. The animals were placed in a modified multiplatform box made of plastic $(50 \mathrm{~cm}$ in length and $30 \mathrm{~cm}$ in height). Ten cylindrical small platforms $(3 \mathrm{~cm}$ in diameter and $10 \mathrm{~cm}$ in length) were positioned on the tank floor. As the platforms will remain $2 \mathrm{~cm}$ above the surface, the container was covered with water $\left(22^{\circ} \mathrm{C}\right) 8$ $\mathrm{cm}$ in depth. Standard laboratory food blocks and a bottle of water were provided to animals to access food and water ad-libitum. The mice were placed on small platforms where they can move. When REM sleep began and after losing muscle tone, the mouse on the platform came into contact with water and awakening. Previous experiments have shown that the large platform used as the standard control reduces 
approximately $80 \%$ of the REM sleep [10]. Therefore, the control mice were housed in their cages separated in the experimental room to be provided in the same environment but benefited their normal sleep period.

\section{Induction of seizures}

To induce seizures, PTZ was injected intraperitoneally (i.p.) at a convulsive dose of $60 \mathrm{mg} / \mathrm{kg}$. After each injection, the mice were individually placed in plexiglass cages for 30 minutes of observation, then the first myoclonic jerk (FMJ), generalized tonic-clonic seizure latencies (GTCS1) and GTCS (GTCSd) duration were recorded. The development of seizure and status epilepticus was evaluated by a behavioral (Racine's Convulsion Scale (RCS)) as follows: $0=$ no convulsion; $1=$ twitching of vibrissae and pinnae; $2=$ motor arrest with more pronounced twitching; $3=$ motor arrest with generalized myoclonic jerks; $4=$ tonic-clonic seizure while the animal remained on its feed; $5=$ tonic-clonic seizure with loss of the righting reflex; and $6=$ lethal seizure. At the end of the experiment, the animals were sacrificed using the decapitation method and their brains were isolated for further evaluation of biochemical parameters.

\section{Biochemical Analysis}

Total Antioxidant Status (TAS) and Total Oxidant Status (TOS) were used as markers for demonstrating the antioxidant and oxidative capacities. The percent ratio of TOS to TAS was accepted as the Oxidative Stress Index (OSI), a marker of the severity of oxidative stress. OSI was calculated as follows: OSI = [(TOS, $\mu \mathrm{mol} \mathrm{H} 2 \mathrm{O} 2 \mathrm{Eq} / \mathrm{mg}$ protein) / (TAS, $\mu \mathrm{mol}$ Trolox Eq/mg protein) $\times 100]$ and evaluated as an indicator of the degree of oxidative stress [11]. Brain tissue samples from each group were homogenized within 10 volumes of the ice-cold homogenization buffer and centrifuged at $12,000 \times \mathrm{g}$ for $10 \mathrm{~min}$ at $4{ }^{\circ} \mathrm{C}$. The supernatant was collected for protein concentration determination by a Bradford protein assay kit (Merck, Germany). Hippocampal antioxidant and oxidant status was determined by using TAS and TOS Assay Kits (Rel Assay Diagnostics ${ }^{\circledR}$ Mega Tip Ltd., Gaziantep, Turkey). Hippocampal GABA and Glutamate levels were analyzed by using gamma aminobutyric acid (GABA) and Glutamate Assay Kits (Rel Assay Diagnostics ${ }^{\circledR}$ Mega Tip Ltd., Gaziantep, Turkey).

\section{Statistical analysis}

The data were presented as the standard deviation of the mean (SEM). The RCS score, FMJ time, duration of GTCS, GTCS latency, and the levels of hippocampal protein were evaluated using a one-way analysis of variance (ANOVA). All statistical analyses were performed using IBM SPSS Statistical software Version 22.0 (IBM, Armonk, NY, USA). A pvalue of less than $5 \%$ was accepted statistically significant.

\section{Results \\ Behavioral measurements}

The effect of REM SD on PTZ-induced seizure parameters is shown in Figure 1 A-D). There was no significant difference between the four groups in the RCS, FMJ, and GTCSl results, but there was a significant increase in the GTCSd results between the REM SD $8+$ PTZ group compared to the PTZ group ( $p<0.05$ ).

Hippocampal TAS, TOS, GABA, and glutamate expressions

The oxidant stress parameters obtained from the hippocampus are given in Figure 2A-C. The TAS levels of REM SD $8+$ PTZ and REM SD $72+$ PTZ groups decreased significantly in the hippocampus compared to the control 
$\operatorname{group}(p<0.001$ and $p<0.05$, respectively). There was a significant decrease in the hippocampal antioxidant capacity in the REM SD $8+$ PTZ group compared to the PTZ group $(p<0.05)$. There was no significant difference in the hippocampal TOS levels between the four groups.

There was a significant increase in OSI levels of the REM SD $8+$ PTZ group compared to the control and the PTZ groups $(p<0.01)$.

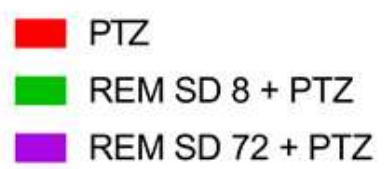

A
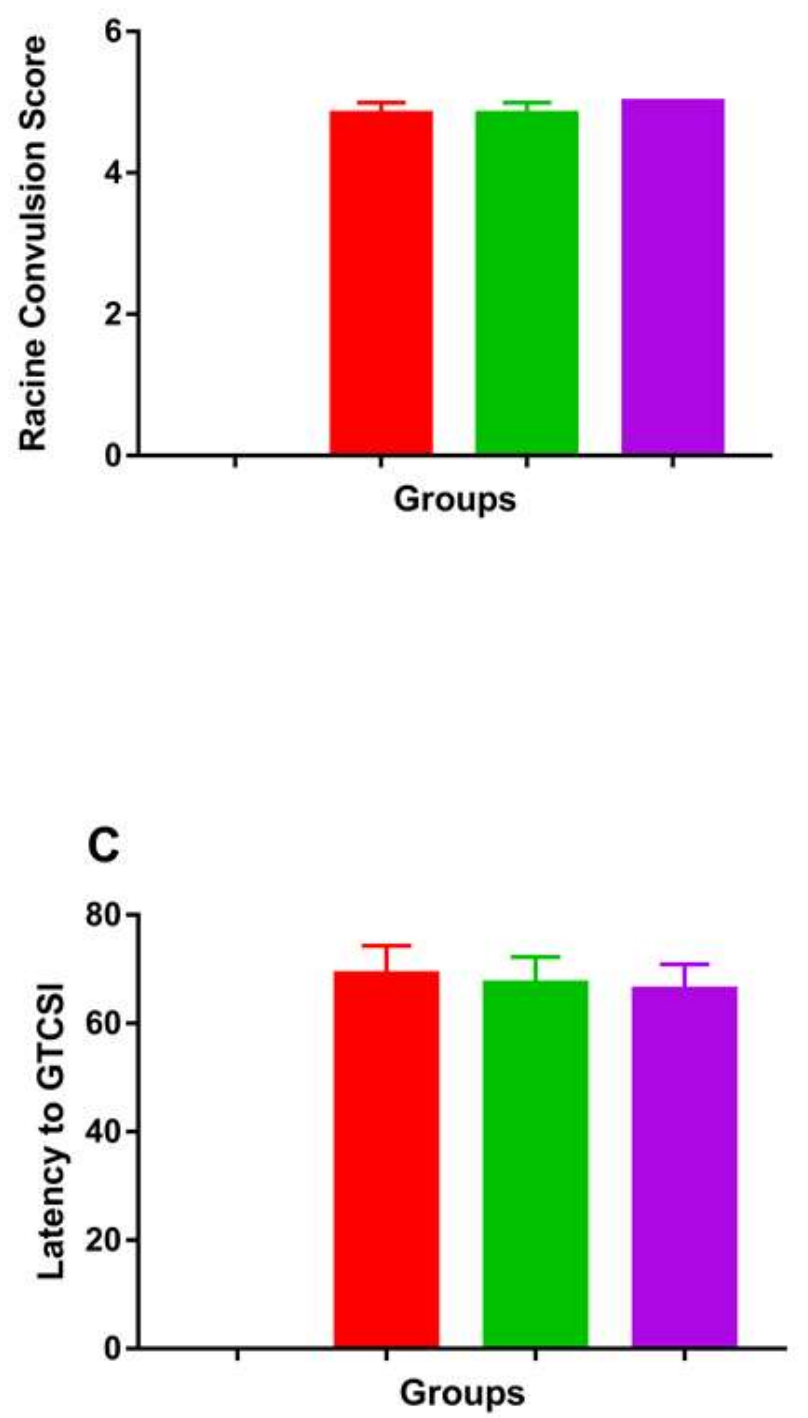

A significant increase in the expression of GABA was found in the REM SD $72+\mathrm{PTZ}$ group $(p<0.05)$.

The increase in the hippocampal glutamate level in the REM SD $8+$ PTZ group was statistically significant $(\mathrm{p}<0.001)$. There was a significant increase in the hippocampal glutamate level in the REM SD 72+PTZ group compared to the control group $(p<0.01)$ (Figure 3A,B).
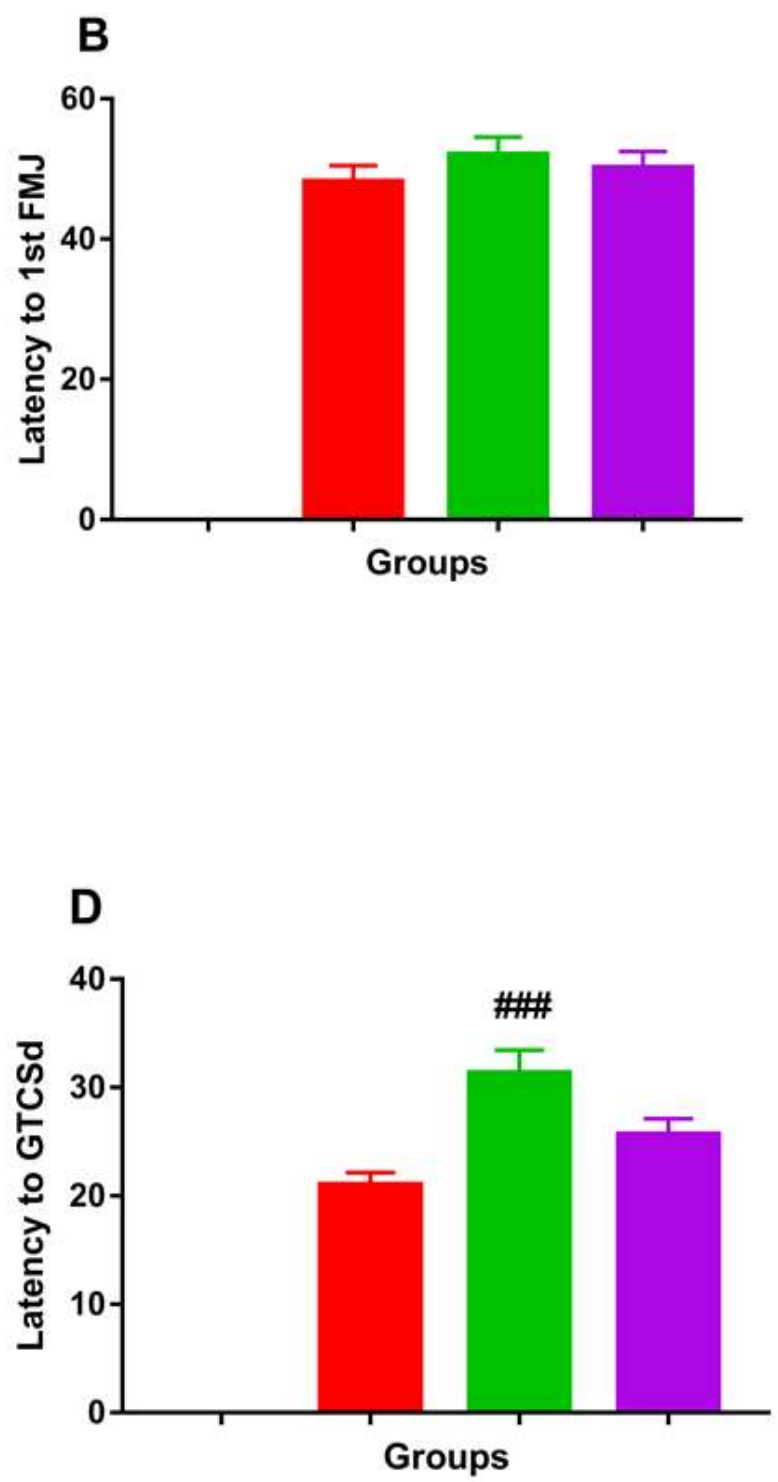

Figure 1A-D. Effects of REM SD on seizures threshold (latency) and duration in PTZ-induced seizures in mice. Data expressed as mean \pm SEM. $\mathrm{n}=6 .{ }^{\# \#} p<0.001$ compared with PTZ group. 


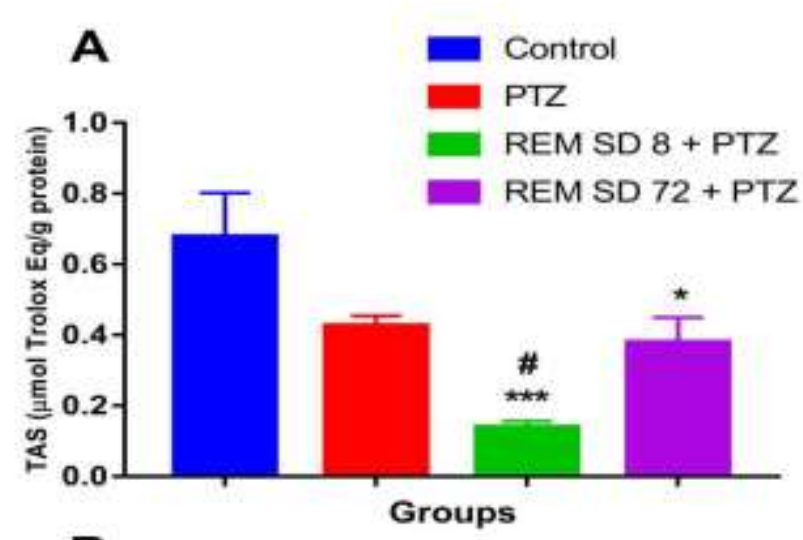

B
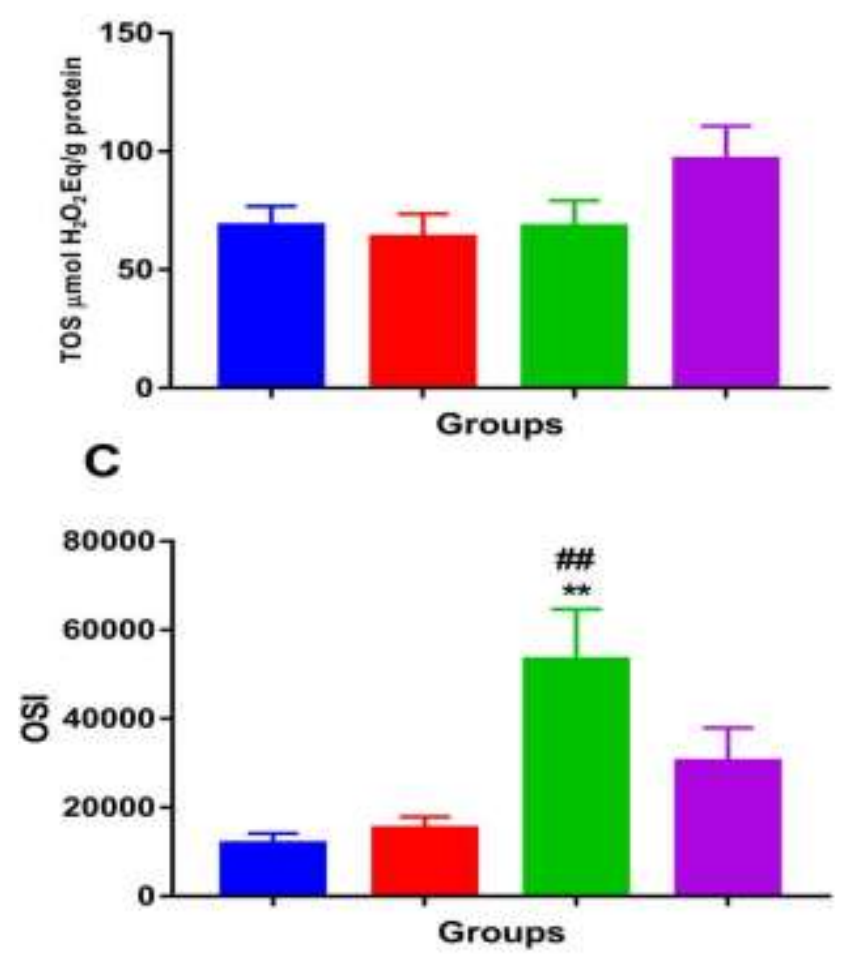

Figure 2A-C. Effects of REM SD and PTZ on hippocampal TAS, TOS, and OSI levels. Data expressed as mean \pm SEM. $\mathrm{n}=6 . * p<0.05$, ** $p<0.01, * * * p<0.001$ compared with Control; \# $p<0.05$, \#\#p<0.01 compared with and PTZ group.

\section{Discussion}

In the present study, we explored the effect of REM SD on seizure susceptibility in the acute epilepsy model. Further, we investigated the effects of PTZ-induced seizures following $8 \mathrm{~h}$ and $72 \mathrm{~h}$ REM SD on excitatory-inhibitory and oxidant-antioxidant balance in the hippocampus. We found that seizure induction after relatively short $(8 \mathrm{~h})$ and long $(72 \mathrm{~h})$ period REM SD differently affect the oxidant
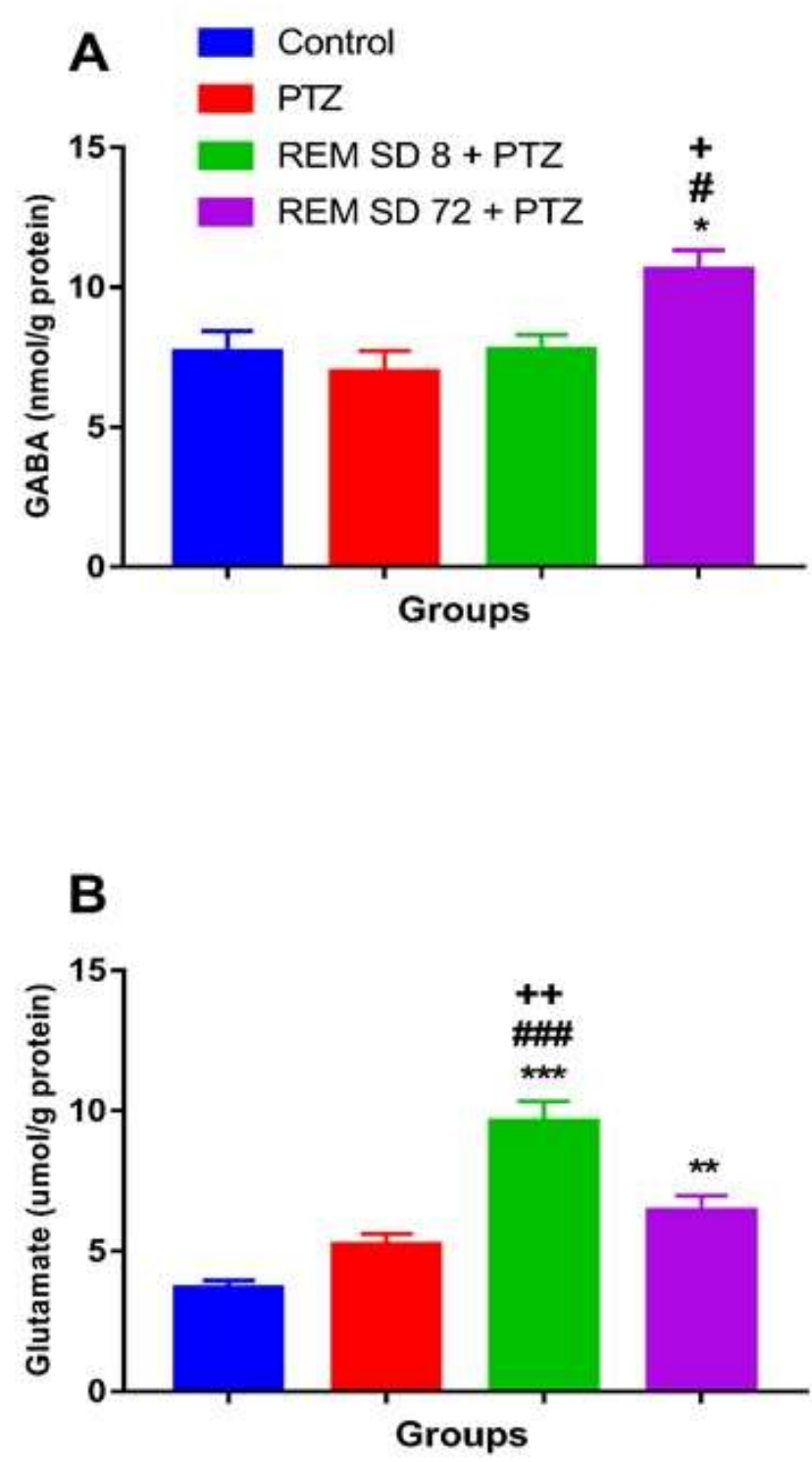

Figure 3A-B. Effects of REM SD and PTZ on hippocampal GABA and Glutamate expressions. Data expressed as mean \pm SEM. $\mathrm{n}=6 .{ }^{*} p<0.05$, $* * p<0.01$, ***p $<0.001$, compared with Control

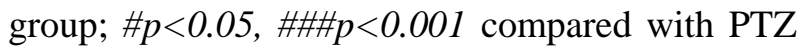
group; $+p<0.05,++p<0.01$ compared with REM SD $72+$ PTZ group.

and antioxidant capacities in the hippocampus. This result was also similar to in hippocampal excitatory and inhibitory tone. The findings of the present study also illustrated the time of REM SD may affect seizure susceptibility. Oxidative stress is a highly disordered metabolic process characterized by increased cellular reactive oxygen species [12]. This event eventually generates an imbalance 
between oxidants and antioxidants. It is well established that both sleep loss and PTZinduced seizure increase oxidative stress in the brain $[13,14]$. We detected only minimal differences in hippocampal TOS levels in this study that did not reach statistical significance. However, our results showed that PTZ-induced seizures after $8 \mathrm{~h}$ and $72 \mathrm{~h}$ REM SD led to a significant decrease in hippocampal TAS levels. It has been assumed that oxygen free radicals accumulate during awake as a result of utilized great amounts of oxygen, and sleep allows the removal of these reactive oxygen species in the brain [13]. Therefore, the wakefulness result from sleep loss disrupts the oxidant and antioxidant balance in the brain. Ramanathan et al. showed that long term (5-11 days) total SD decreased an antioxidative enzyme superoxide dismutase (SOD) activity in the rat hippocampus [15]. In agreement with our results, Suer et al. reported that 21-day REM SD leads to a decrease in the antioxidant defenses in the hippocampus [16]. Moreover, an unexpected but intriguing finding was that the effect of PTZ-induced seizures after $8 \mathrm{~h}$ REM SD was more prominent than $72 \mathrm{~h}$ REM SD on the hippocampal TAS and OSI levels. This unexpected finding can be explained by that the long-term SD may lead to an adaptive reaction of the brain against sleep loss. The present hippocampal GABA results support this hypothesis as its level was increased by PTZ administered following $72 \mathrm{~h}$ REM SD. The PTZ-induced seizures after 8 h REM SD also caused a considerable increase in the hippocampal glutamate (an excitatory transmitter) levels. In line with our results, 6and 12-h total SD resulted in a significant increase in the hippocampal glutamate levels [17]. Taking account that PTZ-induced seizures after the $72 \mathrm{~h}$ REM SD also lead to an increase GABA level in the hippocampus, we think that
REM SD for $8 \mathrm{~h}$ has a more potent effect than REM SD for $72 \mathrm{~h}$ on seizure susceptibility.

In the present study, we also found that the seizures durations were significantly increased after the $8 \mathrm{~h}$ REM sleep loss. The finding implies that REM sleep a critical sleep period for an epileptic phenomenon. In agreement with our results, Marcus et al. concluded that seizures are less common in REM sleep, and this stage of sleep plays a protective role against generalized seizures [8]. However, we could not find a correlation between the REM SD duration and biochemical and behavioral results tested herein. The plausible reason for this could be attributed to the fact that the longer period of REM SD induces a compensatory response that provides resistance to the occurrence of epileptic seizures. It should be noted that the modified multiple platform procedure used in the present study can interfere with the majority of REM sleep, besides it can restrain up to $40 \%$ of NREM sleep [10]. Thus, the NREM sleep deprivation may have also contributed to our results.

\section{Conclusion}

In conclusion, we report that the REM SD may affect seizure susceptibility by altering the balance of the hippocampal oxidantantioxidant, and stimulant and inhibitor. In addition to the acute epilepsy model, investigating seizure susceptibility in a chronic epilepsy model may value further studies in rodent epileptogenesis and SD models.

Conflict of Interest: The authors declare that they have no conflict of interest.

Ethical statement: This experimental study was reviewed and approved by the local ethics committee (Registry Number: 65202830050.04.04-166 dated 09.04.2018). 


\section{Acknowledgements}

Financial support: The work was supported by grants from CUBAP (T-814).

\section{ORCID iD of the author(s)}

Recep Akkaya / 0000-0002-3477-7198

Sebahattin Karabulut /0000-0002-3261-4125

Ahmet Sevki Taskiran / 0000-0002-5810-8415

\section{References}

[1]Perucca E, French J, Bialer M. Development of new antiepileptic drugs: challenges, incentives, and recent advances. Lancet Neurol. 2007; 6(9):793-804.

[2]Cetinkaya A, Demir S, Orallar $\mathrm{H}$ et al. The effects of treadmill exercise on oxidative stress in Mongolian gerbils with penicillininduced epilepsy. Exp Biomed Res. 2018; 1(1):10-16.

[3]Hanci F, Canpolat M, Per $\mathrm{H}$ et al. The relation between antiepileptic drug type and cognitive functions in childhood epilepsy: A prospective observational study. Exp Biomed Res. 2019; 2(2):62-68.

[4]Pitkanen, A, Sutula TP. Is epilepsy a progressive disorder? Prospects for new therapeutic approaches in temporal-lobe epilepsy. Lancet Neurol. 2002; 1(3):173-81.

[5]Baulac M, Pitkanen A. Research priorities in epilepsy for the next decade-A representative view of the European scientific community: Summary of the ILAE Epilepsy Research Workshop, Brussels, 1718 January 2008. Epilepsia. 2009; 50(3):571-83.

[6]Abbott SM, Videnovic A. Chronic sleep disturbance and neural injury: links to neurodegenerative disease. Nat Sci Sleep. 2016:25; 8:55-61.

[7]Scalise A, Desiato MT, Gigli GL, et al. Increasing cortical excitability: a possible explanation for the proconvulsant role of sleep deprivation. Sleep. 2006; 29(12):159598.

[8]Ng M, Pavlova M. Why are seizures rare in rapid eye movement sleep? Review of the frequency of seizures in different sleep stages. Epilepsy Res Treat. 2013;2013:932790.

[9]Soto-Rodriguez S, Lopez-Armas G, Luquin $S$, et al. Rapid Eye Movement Sleep Deprivation Produces Long-Term Detrimental Effects in Spatial Memory and Modifies the Cellular Composition of the Subgranular Zone. Front Cell Neurosci. 2016; 10(132):1-13.

[10] Machado RB, Hipólide DC, Benedito-Silva AA, Tufik S. Sleep deprivation induced by the modified multiple platform technique: quantification of sleep loss and recovery. Brain Res. 2004; 1004(1-2):45-51.

[11] Tufek A, Tokgoz O, Aliosmanoglu I, et al. The protective effects of dexmedetomidine on the liver and remote organs against hepatic ischemia reperfusion injury in rats. Int J Surg. 2013; 11(1):96-100.

[12]Jain S, Webster TJ, Sharma A, et al. Intracellular reactive oxidative stress, cell proliferation and apoptosis of Schwann cells on carbon nanofibrous substrates. Biomaterials. 2013; 34(21):4891-901.

[13]Reimund E. The free-radical flux theory of sleep. Med Hypotheses. 1994; 43(4):231-33.

[14]Rauca C, Zerbe R, Jantze H. Formation of free hydroxyl radicals after pentylenetetrazol-induced seizure and kindling. Brain Res. 1999;847(2):347-51.

[15] Ramanathan L, Gulyani S, Nienhuis R, et al. Sleep deprivation decreases superoxide dismutase activity in rat hippocampus and brainstem. Neuroreport. 2002; 13(11):138790.

[16] Süer C, Dolu N, Artis AS, et al. The effects of long-term sleep deprivation on the long- 
term potentiation in the dentate gyrus and brain oxidation status in rats. Neurosci Res. 2011; 70(1):71-77.

[17] Cortese BM, Mitchell TR, Galloway MP, et al. Region-specific alteration in brain glutamate: possible relationship to risk taking behavior. Physiol Behav. 2010; 99(4):445-50. 